\title{
Variation in Inpatient Rehabilitation Utilization After Hospitalization for Burn Injury in the United States
}

\author{
Nathaniel H. Greene, MD, ${ }^{*}$ Tam N. Pham, MD, FACS, $\uparrow$ \\ Peter C. Esselman, MD, MPT, $\ddagger$ Frederick P. Rivara, MD, MPHS
}

\begin{abstract}
Approximately 45,000 individuals are hospitalized annually for burn treatment. Rehabilitation after hospitalization can offer a significant improvement in functional outcomes. Very little is known nationally about rehabilitation for burns, and practices may vary substantially depending on the region based on observed Medicare posthospitalization spending amounts. This study was designed to measure variation in rehabilitation utilization by state of hospitalization for patients hospitalized with burn injury. This retrospective cohort study used nationally collected data over a 10 -year period (2001 to 2010), from the Healthcare Cost and Utilization Project (HCUP) State Inpatient Databases (SIDs). Patients hospitalized for burn injury $(\mathrm{n}=\mathbf{5 7 , 9 6 8})$ were identified by ICD-9-CM codes and were examined to see specifically if they were discharged immediately after hospitalization (primary endpoint). Both unadjusted and adjusted likelihoods were calculated for each state taking into account the effects of age, insurance status, hospitalization at a burn center, and extent of burn injury by TBSA. The relative risk of discharge to inpatient rehabilitation varied by as much as 6-fold among different states. Higher TBSA, having health insurance, higher age, and burn center hospitalization all increased the likelihood of discharge to inpatient rehabilitation following acute care hospitalization. There was significant variation between states in inpatient rehabilitation utilization after adjusting for variables known to affect each outcome. Future efforts should be focused on identifying the cause of this state-to-state variation, its relationship to patient outcome, and standardizing treatment across the United States. (J Burn Care Res 2014;XXX:00-00)
\end{abstract}

\section{INTRODUCTION}

Burn injury represents a significant health burden in the United States, with an estimated annual incidence of 450,000 cases. Of these, approximately 45,000 individuals are hospitalized annually for burn treatment. ${ }^{1}$ Thanks to many advances in modern burn care, survival has improved dramatically over the past 40 years. $^{2}$ Survivors, however, often

\footnotetext{
From the *Department of Anesthesiology and Pediatrics, Duke University School of Medicine, Durham, North Carolina; tDepartment of Surgery, School of Medicine, University of Washington, Seattle; $\neq$ Department of Rehabilitation Medicine, School of Medicine, University of Washington, Seattle; and SDepartment of Pediatrics, School of Medicine, University of Washington, Seattle.

Address correspondence to Nathaniel Greene, MD, Department of Anesthesiology, DUMC Box 3710, Durbam, North Carolina 27710.

Copyright (C) 2014 by the American Burn Association $1559-047 X / 2014$
}

DOI: $10.1097 / B C R .0000000000000200$ face major physical and psychosocial challenges in order to rehabilitate and reintegrate into society. Thus, increased focus is being placed on transitional care processes to improve long-term outcomes after burns.

Inpatient rehabilitation can provide an important intervention to improve independent living after injury. Burn survivors who qualify for inpatient rehabilitation often have larger injuries, typically $20 \%$ or more TBSA (often defined as "major burn"), and have had lengthy acute care hospitalizations. These patients have complex rehabilitation needs that are best met by further inpatient treatment. ${ }^{3,4}$ Patients who undergo inpatient rehabilitation experience significant functional improvements by discharge from their rehabilitation unit. ${ }^{5,6}$ Yet, little is known about rates of inpatient rehabilitation nationally for burns.

Early transition to inpatient rehabilitation can also decrease acute hospitalization length of stay (LOS), a significant financial incentive for acute care hospitals. In fact, the Centers for Medicare and Medicaid 
Services (CMS) requirements for inpatient rehabilitation facilities (IRF) are favorable to severe burns. These include 1) an active intervention of multiple therapy disciplines, 2) an intensive rehabilitation therapy program (participation for 3 hours/day for 5 days/week), and 3) an expectation of significant benefit for the individual patient. ${ }^{7}$ Despite these guidelines, IRF admissions after burns may also depend on their regional availability, as well as referral and preadmission screening practices. A recent Institute of Medicine report ${ }^{8}$ showed that variations in total Medicare spending by state would decline by $73 \%$ if there were no variation in post-acute care service spending, of which inpatient rehabilitation is a significant portion.

We therefore hypothesized that there is a significant geographic variation in the United States in rates of inpatient rehabilitation use after major burns.

\section{METHODS}

\section{Overview of Study Design}

This retrospective cohort study was conducted to examine differences in discharge disposition of all patients admitted to a hospital with diagnosis of a burn injury. The Healthcare Cost and Utilization Project (HCUP) State Inpatient Database (SID) was used to identify all relevant hospitalizations in reporting states and subsequent outcomes. Discharge to inpatient rehabilitation among inpatients who were discharged alive served as the primary outcome of interest. State-specific relative risk estimates were generated using multivariable models to best describe the effect each state had on both our primary outcome. Human subjects' approval was not required for analysis of these publically available datasets without identifiable information.

\section{Data Sources}

The SID is a set of hospital databases from data organizations in participating states, and contains the universe of the state inpatient discharge abstracts, translated into a uniform format to facilitate multistate comparisons and analyses. The SID contains a core set of clinical and nonclinical information on all patients, regardless of payer, including persons covered by Medicare, Medicaid, private insurance, and the uninsured. A subset of the SID was used for this analysis: 12 states from 2001 (CO, FL, IA, MD, $\mathrm{MA}, \mathrm{NJ}, \mathrm{NY}, \mathrm{NC}, \mathrm{OR}, \mathrm{UT}, \mathrm{WA}$, and WV), 17 states from 2004 (AZ, CO, FL, IA, KY, MD, MA, NV, NJ, $\mathrm{NY}, \mathrm{NC}, \mathrm{OR}, \mathrm{UT}, \mathrm{VT}, \mathrm{WA}, \mathrm{WV}$, and WI), 15 states from 2007 (AR, AZ, CO, FL, IA, KY, MD, NV, NJ,
NC, OR, UT, VT, WA, and WV), and 18 states from 2010 (AR, AZ, CO, FL, IA, KY, MD, MS, NC, NJ, NV, NY, OR, UT, VT, WA, WI, and WV). These states were chosen because of the availability of data to the investigators. A significant number of states did not participate in the SID in the years examined, but most that did were included in this analysis. It should be noted that this database treats rehabilitation units of the same hospital building as different hospitals, and thus also captures discharge to a rehabilitation unit within the same hospital.

\section{Sampling Methods}

We identified all patients hospitalized with burns (ICD-9-CM codes 940-949, with all available decimals) in participating states during the selected years. All available discharge codes were sorted into seven mutually exclusive discharge groups: home, skilled nursing facility (SNF), inpatient rehabilitation, transfer to another acute care hospital, psychiatric hospital, hospice, and death. We excluded patients with a discharge disposition indicating "transfer to another acute care hospital" to avoid double counting. Three states did not provide data on discharge to rehabilitation; California did not have such a code in their dataset while Maine and Rhode Island had such a code, but it never appeared in data from either state.

\section{Independent Variables of Interest}

In addition to our main independent variable of interest (state), we were also interested in the independent contributions of age (as a continuous variable), insurance status (government, private, or uninsured), burn injury severity by TBSA decile (derived directly from the provided ICD-9-CM code, when available), sex, and hospitalization in an ABA-verified burn center.

\section{Missing Data}

Data were missing from the SID in $36.8 \%$ of cases for TBSA severity, $0.1 \%$ for insurance status, $1.0 \%$ for age, $0.02 \%$ for LOS, $0.7 \%$ for sex, and $0.1 \%$ for discharge disposition. Cases with missing data for insurance status, age, LOS, sex, and discharge disposition were dropped. Multiple imputation method was used to predict missing TBSA severity by decile, since this produces less biased results than if such cases were dropped from the analyses. Our model assumes that the data in our sample are missing-atrandom and thus can be appropriately analyzed using multiple imputation methods. ${ }^{9}$ Accordingly, we used Stata's MI procedure to generate 10 imputation datasets with predicted values for missing covariates 
using multiple chained equations and hospitalization state, discharge disposition, age, LOS, sex, and TBSA severity as predictors. In subsequent analyses, Stata's MI estimate routine was used with regression functions to analyze the data and make appropriate adjustments to the reported standard errors.

\section{Multivariable Regression Analysis}

The primary outcome of interest, discharge to inpatient rehabilitation (out of total live discharges) was examined in multiple regression analyses. Independent variables included state of hospitalization, insurance status, age, and TBSA severity. Patients with LOS less than 2 days were excluded from the multivariable analysis because these patients were unlikely to need inpatient rehabilitation. Multivariable Poisson regression with robust standard error estimates and clustering by hospital was used for our outcome, given the non-rare occurrence of our outcome of interest. Although Poisson regression is typically used for count outcomes, robust Poisson regression is an alternative to logistic regression for non-rare outcomes $(>10 \%)$ overall ${ }^{10}$ or among subgroups of interest. ${ }^{11}$ States with the lowest adjusted risk estimates were selected as referent states for ease of communication. All analyses were performed using Stata Intercooled 12 (StataCorp LP, College Station, TX).

\section{RESULTS}

\section{Patient Characteristics}

There were 57,968 patients hospitalized with burn

Tl injury in the years and states examined (Table 1). In general, patients were men $(63.7 \%)$, had 0 to $10 \%$ TBSA $(72.6 \%)$, were of a wide variety of ages, had government-based insurance $(59 \%)$, and were discharged to home $(87.4 \%)$.

\section{Unadjusted Outcomes}

For the entire cohort, there were significant differences by state in the proportion of discharges to inpatient rehabilitation among live discharges

T2 (Table 2). This proportion ranged from $1.2 \%$ in Mississippi, Washington, and West Virginia, to $8.4 \%$ in Massachusetts. The proportion also varied by TBSA decile from $1.2 \%$ in the 0 to $10 \%$ decile to a high of $21.3 \%$ in the 60 to $70 \%$ TBSA decile. Type of insurance was also a factor with $0.5 \%$ of patients without insurance discharged to inpatient rehabilitation compared to $2.7 \%$ of government-based insurance. Men and women exhibited similar rates of discharge $(2.3 \%$ and $2.2 \%$, respectively). Burn centers had a higher proportion of discharges to rehabilitation at $3.2 \%$ compared to $1.9 \%$ for non-burn centers.

\section{Adjusted Outcomes}

Using a multivariable Poisson regression model, a patient hospitalized in Maryland was 6.6 times more likely to be discharged to inpatient rehabilitation compared to an otherwise similar patient hospitalized in Washington (Figure 1; Table 2). A patient with $>40 \%$ TBSA was more than 10 times as likely to be discharged to inpatient rehabilitation than those with the smallest burns. Someone without insurance was 0.25 times as likely to receive inpatient rehabilitation care and someone hospitalized at a burn center was approximately twice as likely to be discharged to inpatient rehabilitation. There was not a significant difference by sex, but there was a significant difference of $2 \%$ higher likelihood of discharge to rehabilitation for every year increase in age.

\section{Differences Among High and Low Rehabilitation Utilization States}

We specifically investigated differences in disposition status for the two states with the highest relative risk

Table 1. Characteristics of patients hospitalized with any burn injury in the select states of the United States in 2001, 2004, 2007, and $2010(\mathrm{n}=57,968)$

\begin{tabular}{lcc}
\hline Characteristic & Mean & SD \\
\hline Age (years) & 38.8 & 24.8 \\
Characteristic & Number & $(\%)$ \\
Female (\%) & 21,036 & $(36.3)$ \\
TBSA burn injury & & \\
0-10\% & 42,064 & $(72.6)$ \\
10-20\% & 9,885 & $(17.1)$ \\
20-30\% & 2,927 & $(5)$ \\
30-40\% & 1,235 & $(2.1)$ \\
$40-50 \%$ & 646 & $(1.1)$ \\
$50-60 \%$ & 391 & $(0.7)$ \\
60-70\% & 250 & $(0.4)$ \\
$70-80 \%$ & 156 & $(0.3)$ \\
80-90\% & 176 & $(0.3)$ \\
90-100\% & 323 & $(0.6)$ \\
Insurance & & \\
Government & 34,190 & $(59)$ \\
Private & 16,682 & $(28.8)$ \\
Uninsured & 7,180 & $(12.4)$ \\
Disposition & & \\
Home & 50,677 & $(87.4)$ \\
SNF & 4,134 & $(7.1)$ \\
Inpatient rehab & 1,071 & $(1.8)$ \\
Psychiatric & 284 & $(0.5)$ \\
Hospice & 201 & $(2.9)$ \\
Died & 1,685 & \\
\hline
\end{tabular}


Table 2. Unadjusted proportion and adjusted likelihood of rehabilitation for patients hospitalized with burn injury in the select states of the United States in 2001, 2004, 2007 , and 2010

\begin{tabular}{|c|c|c|c|c|}
\hline & $\mathbf{N}$ & Crude \% & aRR & $95 \% \mathrm{CI}$ \\
\hline \multicolumn{5}{|l|}{ State } \\
\hline Arkansas & 953 & 1.6 & 1.41 & {$[0.36,5.6]$} \\
\hline Arizona & 2932 & 1.9 & 1.82 & {$[0.82,4.02]$} \\
\hline Colorado & 1722 & 4.2 & 3.40 & {$[2.19,5.28]$} \\
\hline Florida & 8222 & 1.3 & 1.34 & {$[0.81,2.22]$} \\
\hline Iowa & 1432 & 4.9 & 3.78 & {$[1.64,8.69]$} \\
\hline Kentucky & 1808 & 2.7 & 2.76 & {$[1.46,5.21]$} \\
\hline Massachusetts & 1849 & 8.4 & 6.15 & {$[4.35,8.71]$} \\
\hline Maryland & 2338 & 5.4 & 6.63 & {$[4.05,10.85]$} \\
\hline Mississippi & 589 & 1.2 & 1.35 & {$[0.49,3.76]$} \\
\hline Nevada & 816 & 4.2 & 1.48 & {$[0.66,3.32]$} \\
\hline New Jersey & 2967 & 1.8 & 1.74 & {$[1.01,3]$} \\
\hline New York & 8480 & 1.4 & 4.80 & {$[3.14,7.36]$} \\
\hline North Carolina & 4296 & 1.6 & 2.34 & {$[1.51,3.62]$} \\
\hline Oregon & 1262 & 1.9 & 1.51 & {$[1.06,2.15]$} \\
\hline Utah & 1118 & 1.9 & 1.44 & {$[0.92,2.25]$} \\
\hline Vermont & 171 & 1.8 & 1.88 & {$[0.98,3.58]$} \\
\hline Washington & 2688 & 1.2 & 1.00 & Referent \\
\hline Wisconsin & 1285 & 2.6 & 2.68 & {$[1.64,4.38]$} \\
\hline West Virginia & 1217 & 1.2 & 1.26 & {$[0.71,2.23]$} \\
\hline \multicolumn{5}{|l|}{ TBSA decile } \\
\hline $0-10 \%$ & 33081 & 1.2 & 1.00 & Referent \\
\hline $10-20 \%$ & 8627 & 2.8 & 2.44 & {$[1.97,3.02]$} \\
\hline $20-30 \%$ & 2501 & 5.6 & 4.85 & {$[3.72,6.32]$} \\
\hline $30-40 \%$ & 959 & 10.9 & 8.88 & {$[6.85,11.52]$} \\
\hline $40-50 \%$ & 438 & 15.0 & 12.15 & {$[8.87,16.65]$} \\
\hline $50-60 \%$ & 216 & 17.0 & 14.84 & {$[9.41,23.4]$} \\
\hline $60-70 \%$ & 123 & 21.3 & 17.47 & {$[11.59,26.33]$} \\
\hline $70-80 \%$ & 60 & 20.2 & 15.43 & {$[8.64,27.54]$} \\
\hline $80-90 \%$ & 53 & 17.5 & 15.21 & {$[7,33.05]$} \\
\hline $90-100 \%$ & 88 & 17.0 & 12.62 & {$[6.76,23.59]$} \\
\hline \multicolumn{5}{|l|}{ Type of insurance } \\
\hline Government & 27778 & 2.7 & 1.00 & Referent \\
\hline Private & 13092 & 2.1 & 0.92 & {$[0.79,1.07]$} \\
\hline Uninsured & 5275 & 0.5 & 0.25 & {$[0.16,0.39]$} \\
\hline \multicolumn{5}{|l|}{ Age } \\
\hline Per year & 46145 & - & 1.02 & {$[1.02,1.03]$} \\
\hline \multicolumn{5}{|l|}{ Sex } \\
\hline Male & 29063 & 2.3 & 1.00 & Referent \\
\hline Female & 17082 & 2.2 & 1.02 & {$[0.91,1.14]$} \\
\hline \multicolumn{5}{|l|}{ Burn center } \\
\hline Non-burn center & 33114 & 1.9 & 1.00 & Referent \\
\hline Burn center & 13031 & 3.2 & 2.03 & {$[1.44,2.85]$} \\
\hline
\end{tabular}

of inpatient rehabilitation discharge (MA, MD, designated "high referral") compared to the lowest two states (WA, WV, designated "low referral"). High referral states had a discharge rate of $26 \%$ to IRF compared to $8 \%$ in WA and WV. Rates of discharges to home were $64 \%$ in high referral states compared to $83 \%$ in low referral states. Skilled nursing facility discharge rates were $9 \%$ in high referral states compared to $9 \%$ in low referral states (Table 3 ).

\section{DISCUSSION}

Current CMS guidelines for inpatient rehabilitation are well suited for burns, as many survivors require intensive treatment to properly transition toward independent living. Increased age, hospitalization at a burn center, and possessing health insurance all increased the likelihood of an inpatient rehabilitation stay following hospitalization for a burn injury, while a higher TBSA generally demonstrated higher rehabilitation utilization until TBSA surpassed $70 \%$, when it began to decrease again. While larger surface area injuries naturally entail more rehabilitation needs, this observation may reflect increasing comorbidity as a hindrance to qualify for rehabilitation placement and instead patients are either discharged to a skilled nursing facility or stay in the hospital long enough to be discharged home. Health insurance carriage is a modifiable patient factor, especially following health care reform. There was no evidence to suggest a difference in rehabilitation utilization between private insurance and government-sponsored insurance. As such, an act as simple as signing up a hospitalized burn patient for health insurance may enable a rehabilitation stay that could improve that patient's overall outcome.

Our principal finding was of significant variation by state in the use of inpatient rehabilitation after hospitalization in patients with burn injury. After adjustment for confounders using multivariable techniques, this variation persisted. The degree of differences between states measured in this study strongly support that these findings are not due to chance. Unmeasured factors that may be responsible for these differences include local physician practice preferences as well as rehabilitation bed availability and charitable funding for rehabilitation after a burn injury. Depending on the institution, IRF may be located in the same or adjacent building, or at a remote distance from the referring acute care facilities. The level of collaboration, availability of rehabilitation providers to make screening visits to hospitalized burn patients could also factor in rates of referral. Burn centers may have established protocols for inpatient rehabilitation referral following acute care, whereas hospitals that occasionally care for burn patients may not. This would argue for increased regionalization of care, as burn center treatment results in higher rehabilitation utilization.

Among states with low rates of referral to IRF, WA and WV hospitals had higher proportions of home discharge, whereas their rates of nursing facilities referrals 


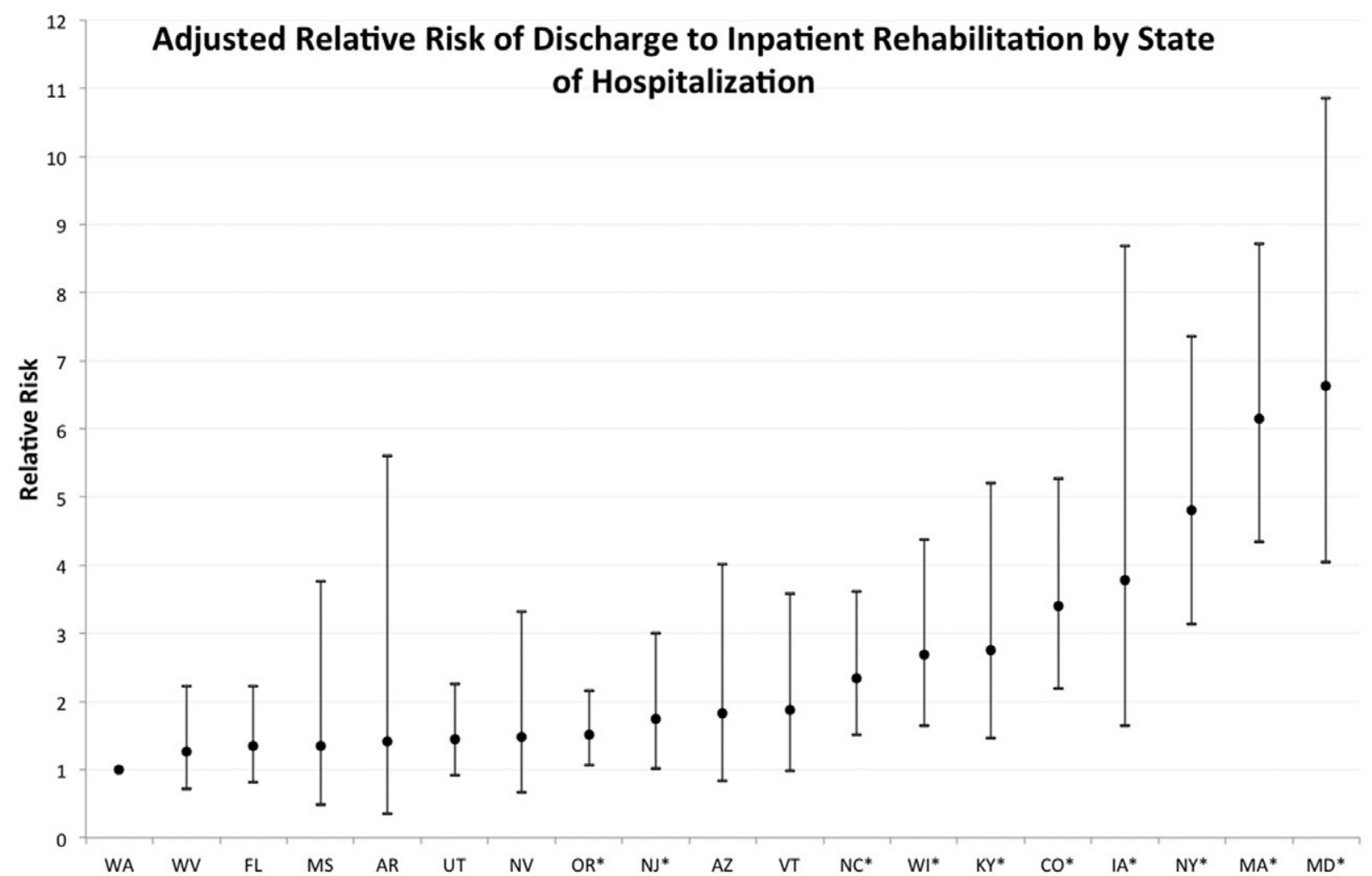

Figure 1. Multivariate Poisson regression point estimates for relative risk by state of hospitalization with $95 \%$ confidence interval estimates, ${ }^{*} P<.05$.

remained comparable to those in MD and MA. We thus postulate that centers in low referral states might have provided sufficient rehabilitative services as part of the acute burn care treatment to achieve home discharges in a high proportion of their patients. This strategy, however, is not a cost-efficient alternative because it tends to lengthen acute care LOS instead of referral to less expensive IRF. In light of these results, we are currently working at our institution to strengthen the partnership between burn care and rehabilitation teams, to devise early screening criteria in order to increase our own rates of inpatient rehabilitation referral.

The limitations of this study should be considered when interpreting these results. While the SID databases include a very large proportion of hospitalizations in the United States, they do not automatically include hospitalizations that were not billed. Participation from hospitals that do not bill for care is voluntary and is not $100 \%$. For example, only one of the two Shriners hospitals within the states studied, which care for a significant number of pediatric burn injuries, is included in the dataset. We also used ICD-9 codes to identify the presence of a burn injury and severity of a burn injury, and although severity was missing in a minority of cases, our statistical methods appropriately account for that fact assuming that the relationship between the likelihood of data being missing and severity of burn injury is random. Even if there is such a relationship, there is no reason to believe that this relationship would vary between states.

Although measuring inpatient rehabilitation referral mainly reflects a process of care, we believe that

AQ6 Table 3. High/low rehabilitation utilization state comparison

\begin{tabular}{lccccccc}
\hline & Home & SNF & Rehab & Psych & Hospice & Died & Totals \\
\hline Massachusetts & 129 & 17 & 63 & 2 & 0 & 39 & 250 \\
Maryland & 133 & 20 & 41 & 2 & 0 & 54 & 249 \\
Washington & 186 & 17 & 25 & 2 & 1 & 54 & 286 \\
West Virginia & 110 & 15 & 4 & 0 & 2 & 15 & 145 \\
\hline
\end{tabular}

${ }^{*}$ Limited to length of stay $\geq 2$ and $T B S A \geq 20 \%$. 
this transitional care intervention leads to measurable improvements in outcomes, especially in vulnerable patients. In the case of older patients, discharge to a nursing facility is a much less appealing alternative. ${ }^{12}$ As such, future work should address whether increasing referral to inpatient rehabilitation will improve long-term survival, decrease readmission rates, and increase the likelihood of return to independent living in older adults with burns. We also recommend a closer examination of health policy and structure of burn care delivery that may serve as the basis of this variation in each state. This includes hospitals in each state examining their own trends and ensuring they are utilizing post-hospitalization rehabilitation appropriately. It is the authors' hope that if this study were done 10 years later, less variation would be observed between states as a result of appropriate rehabilitation care utilization after hospitalization for a burn injury. Establishing national guidelines for rehabilitation care after a burn injury may serve as the starting point to decrease this variation.

\section{AQ8 ACKNOWLEDGMENTS}

This work was supported by funds from the National Institute on Disability and Rehabilitation Research in the Office of Special Education and Rehabilitative Services in the U.S. Department of Education (H133Al20024).

\section{REFERENCES}

1. American Burn Association 2012 Fact Sheet; available from http://www.ameriburn.org/resources_factsheet.php.

2. Engrav LH, Heimbach DM, Rivara FP, et al. Harborview burns - 1974 to 2009. PLoS One 2012;7:e40086.

3. Ward EC, Uriarte M, Sppath B, Conroy AL, Sppatht B. Duration of dysphagic symptoms and swallowing outcomes after thermal burn injury. J Burn Care Rehabil 2001;22:44153; discussion 440-1.

4. Schneider JC, Bassi S, Ryan CM. Barriers impacting employment after burn injury. J Burn Care Res 2009;30:294-300.

5. Sliwa JA, Heinemann A, Semik P. Inpatient rehabilitation following burn injury: patient demographics and functional outcomes. Arch Phys Med Rehabil 2005;86:1920-3.

6. Spires MC, Bowden ML, Ahrns KS, Wahl WL. Impact of an inpatient rehabilitation facility on functional outcome and length of stay of burn survivors. J Burn Care Rehabil 2005;26:532-8.

7. Center for Medicare and Medicaid Services: Inpatient Rehabilitation Fact Sheet, 2014, available from http:// www.cms.gov/Outreach-and-Education/MedicareLearning-Network-MLN/MLNProducts/downloads / Inpatient_Rehab_Fact_Sheet_ICN905643.pdf.

8. Newhouse JP, Garber AM. Geographic variation in health care spending in the United States: insights from an Institute of Medicine report. JAMA 2013;310:1227-8.

9. Greenland S, Finkle WD. A critical look at methods for handling missing covariates in epidemiologic regression analyses. Am J Epidemiol 1995;142:1255-64.

10. Zou G. A modified poisson regression approach to prospective studies with binary data. Am J Epidemiol 2004;159:702-6.

11. Cummings $P$. The relative merits of risk ratios and odds ratios. Arch Pediatr Adolesc Med 2009;163:438-45.

12. Davidson GH, Hamlat CA, Rivara FP, Koepsell TD, Jurkovich GJ, Arbabi S. Long-term survival of adult trauma patients. JAMA 2011;305:1001-7.

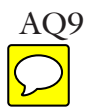




\section{AUTHOR QUERIES}

\section{AUTHOR PLEASE ANSWER ALL QUERIES}

AQ1-All the headings within the abstract have been deleted to make it an unstructured abstract. Please confirm if this is fine.

AQ2-Please define "ABA."

AQ3- "Multiple imputation" has been changed as "Multiple imputation method" in the sentence "Multiple imputation method was used...". Please check if this is fine.

AQ4-Please define "MI."

AQ5-Please check if Table 2 is presented correctly.

AQ6-The footnote indicator " $*$ " is not cited in the table body of Table 3. Please check.

AQ7-The abbreviation "LOS" has been expanded as "length of stay" in the footnote of Table 3. Please check if this is fine.

AQ8-Please check if the Acknowledgments section here is set correctly.

AQ9-Please provide accessed date for the website address and year of publication in ref. 1. AQ10-Please provide accessed date for the website address in ref. 7. 